Klin. Pädiat. 197 (1985) 44-49

(C) F. Enke Verlag Stuttgart

\title{
Sonographischer Nachweis von Hirntumoren im Säuglingsalter
}

\author{
K.H. Deeg, H. Segerer, D. Harms
}

Kinderklinik und Poliklinik der Universität Erlangen-Nürnberg (Direktor: Prof. Dr. K. Stehr)

\section{Zusammenfassung}

Bei 3 Kindern im Alter von 1 Tag bis 5 Monaten wurde mit der zweidimensionalen Schädelsonographie durch die offene Fontanelle ein Hirntumor nachgewiesen. Die Tumore waren im Ultraschall durch ihre echodichte Struktur und ihre gute Abgrenzbarkeit von der Umgebung gekennzeichnet. Bei 2 Kindern lag ein infratentorieller Tumor vor. Das Medullablastom sowie auch der unklassifizierbare neuroektodermale Tumor hatten die Entwicklung eines Hydrozephalus internus verursacht, der sich sonografisch ebenfalls gut darstellen ließ. Beide Kinder waren inoperabel und verstarben durch Versagen der zentralen Regulationen im Alter von 3 und 5 Monaten. Bei dem 3. Kind lag ein Plexuspapillom vor, das einen Hydrozephalus hyersekretorius verursacht hatte. Nach operativer Entfernung des Tumors bildete sich der Hydrozephalus spontan zurück.

Vergleichende Untersuchungen mit der kranialen Computertomographie und dem Autopsiebefund zeigten, daß durch die Sonographie die Tumore hinsichtlich ihrer Lokalisation, Größe und Abgrenzbarkeit exakt erfaßt wurden.

\section{Ultrasonographic Demonstration of Brain Tumors in Infancy}

Using the open fontanelle as an acustic window brain tumours were diagnosed by gray scale ultrasonography in 3 infants aged 1 day to 5 months. The tumours were charakterized by their echodense structure and their good delimination from the surrounding brain.

In 2 children the tumour was localized infratentorially (medulloblastoma and unclassified neuroectodermal tumour) and had caused an occlusive hydrocephalus. Both children died aged 3 and 5 months in central nervous dysregulation. One child suffered from plexus papilloma which had caused a hypersecretory hydrocephalus. After resection of the tumour the hydrocephalus decreased without any further treatment. Comparison with axial computed tomography and autopsy findings showed, that gray scale ultrasonography is equally efficient in diagnosing brain tumours and associated hydrocephalus.

\section{Einleitung}

Leistungsfähige Sektorscangeräte ermöglichen die sonographische Untersuchung des Säuglingsgehirns durch die offene Fontanelle als sogenanntes akustisches Fenster $(1,3,9$, 14, 20, 21, 25, 26). Bei kleiner Ankoppelungsfläche des Schallkopfes und großem Bildausschnitt können die anatomischen Gegebenheiten des Säuglingsschädels sehr gut genutzt werden. Mit der zweidimensionalen Schädelsonographie lassen sich wichtige intrakranielle Strukturen wie Großhirn, Kleinhirn, Hirnstamm, Ventrikelsystem mit Plexus chorioi- dei, Stammganglien, Corpus callosum und große intrakranielle Gefäße (Arterien, Sinus) exakt erfassen. In gleicher Weise können die wichtigsten pathologischen Veränderun. gen sicher dargestellt werden, wie vergleichende Untersuchungen mit der Computertomographie gezeigt haben $(3,21$, $25,27)$. So sind Hirnblutungen $(1,3-9,11,14,25)$, die verschiedenen Hydrozephalusformen $(1,3-9,11,14,21$, $25,27)$, Subduralergüsse $(4,11)$, das Hirnödem und angeborene Mißbildungen des ZNS wie das Dandy-Walker-Syndrom (11), das Arnold-Chiari-Syndrom, auch Zysten des Septum pellucidum und Agenesien des Corpus callosum (4), Enzephalozelen (4) sowie arteriovenöse Aneurysmen der Vena Galeni $(26,28)$ eindeutig nachweisbar.

Aufgrund ihrer Seltenheit im Säuglingsalter ist bisher über den Nachweis von Hirntumoren mit der zweidimensionalen Sonographie nur wenig berichtet worden (13).

\section{Patienten und Methode}

Bei ca. 3000 Ultraschalluntersuchungen von Säuglingsgehirnen in den Jahren 1980-1983 wurde bei 3 Kindern ein Hirntumor diagnostiziert und die sonographischen Befunde mit der kranialen Computertomographie (CT) sowie in 2 Fällen mit dem Autopsiebefund verglichen. Alle 3 Kinder wurden mit dem Sonographiesystem RA-1 (Fa. Siemens) untersucht. Das Gerät ist mit einem 3,5- und $5 \mathrm{MHz}$ Schallkopf mit wahlweiser Möglichkeit zum Compoundscan oder schnellen B-Bild ausgestattet (Sektor $60^{\circ}$ bzw. $100^{\circ}$ ). Bei allen Kindern wurden Koronar-, Sagittal- und Parasagittalschnitte durch die offene Fontanelle durchgeführt. Die sonographischen Verlaufskontrollen erfolgten in wöchentlichen Abständen.

\section{Kasuistik}

Patient 1 (K. T.): Geburt in der 39. Schwangerschaftswoche durch Sectio wegen drohender intrauteriner Asphyxie. Apgar-Score 0/3/3. Sofortige Intubation und maschinelle Beatmung wegen zentraler Ateminsuffizienz.

Befund: $2600 \mathrm{~g}$ schweres, weibliches Neugeborenes ohne Spontanaktivität. Makrozephale Kopfform mit vorgewölbter gespannter Fontanelle. Kopfumfang $37 \mathrm{~cm}$ (97. Percentile). Anisokorie mit lichtstarrer rechter Pupille. Tonischklonische, generalisierte Krampfanfälle.

\section{Diagnostik}

a Sonographie: Gut von der Umgebung abgrenzbare, echodichte, intrazerebrale Raumforderung okzipitotemporal 
Tab. 1 Daten der Patienten

\begin{tabular}{|c|c|c|c|}
\hline & Patient 1 & Patient 2 & Patient 3 \\
\hline \multirow{4}{*}{ Symptome } & 1. Ateminsuffizienz & 1. Erbrechen & \multirow{4}{*}{$\begin{array}{l}\text { 1. Erbrechen } \\
\text { 2. hydrozephale Zeichen } \\
\text { - makrozephale Kopfform } \\
\text { - gespannte, vorgewölbte } \\
\text { Fontanelle } \\
\text { - Sonnenuntergangs- } \\
\text { phänomen }\end{array}$} \\
\hline & $\begin{array}{l}\text { 2. hydrozephale Zeichen } \\
\text { - makrozephale Kopfform } \\
\text { - gespannte, vorgewölbte } \\
\text { Fontanelle }\end{array}$ & $\begin{array}{l}\text { 2. hydrozephale Zeichen } \\
\text { - makrozephale Kopfform } \\
\text { - gespannte, vorgewölbte } \\
\text { Fontanelle }\end{array}$ & \\
\hline & $\begin{array}{l}\text { 3. ophthalmolog. Befunde } \\
\text { - Anisokorie } \\
\text { - lichtstarre Pupille re. }\end{array}$ & $\begin{array}{l}\text { 3. ophthalmolog. Befunde } \\
\text { - fehlende Fixation } \\
\text { - Anisokorie } \\
\text { - fichtstarre Pupille li. }\end{array}$ & \\
\hline & 4. tonisch-klonische Krampfanfälle & 4. tonisch-klonische Krampfanfälle & \\
\hline Alter bei Diagnose: & $1 \mathrm{Tag}$ & $41 / 2$ Monate & 2 Monate \\
\hline Kopfumfang bei Diagnose: & $37 \mathrm{~cm}$ (97. Percentile) & $47 \mathrm{~cm}$ (97. Percentile) & $41 \mathrm{~cm}$ (90. Percentile) \\
\hline Lokalisation: & infratentoriell & infratentoriell & supratentoriell \\
\hline Hydrozephalus: & okklusus & okklusus & hypersekretorius \\
\hline Therapie: & keine & keine & Resektion \\
\hline Verlauf: & $\begin{array}{l}\text { Tod mit } 3 \text { Monaten } \\
\text { zentrale Ateminsuffizienz }\end{array}$ & $\begin{array}{l}\text { Tod mit } 5 \text { Monaten } \\
\text { zentrale Ateminsuffizienz. }\end{array}$ & $\begin{array}{l}\text { Rückbildung des Hydrozepha. } \\
\text { lus nach Tumorresektion }\end{array}$ \\
\hline Histologie: & $\begin{array}{l}\text { unklassifizierbarer neuro. } \\
\text { ektodermaler Tumor }\end{array}$ & Medulloblastom & Plexuspapillom \\
\hline
\end{tabular}

links (Abb. 1 und 3), die zur Verlagerung der Mittelstrukturen nach rechts geführt hat. Nach lateral verlagerter rechter Seitenventrikel, 3. und 4. Ventrikel nach ventral verlagert. Der Tumor ist nicht eindeutig vom Kleinhirn abgrenzbar (Abb. 1 und 2).

b Computertomographie: Ausgedehnter intrazerebraler Tumor okzipitotemporal links (Abb. 4).

Verlauf: Aufgrund der Größe sowie der Lokalisation des Tumors und seiner infausten Prognose wurde von einer Thera- pie abgesehen. Im weiteren Verlauf traten tonisch-klonische Krampfanfälle und die Entwicklung eines progressiven Hydrozephalus internus (Abb. 2-4) mit zystisch-wabiger Umwandlung beider Großhirnhemisphären (Abb. 3 und 4) auf. Das Kind starb im Alter von 12 Wochen durch Versagen der zentralen Regulationen.

Sektionsbefund: $8,5 \times 5 \times 4 \mathrm{~cm}$ große Tumormasse der hinteren Schädelgrube, die nach rostral bis zum Septum pellucidum und nach kaudal bis in den linken Okzipitalpol reichte. Nahezu vollständige Destruktion des Thalamus und Corpus callosum. Hydrozephalus internus des re. Sei-
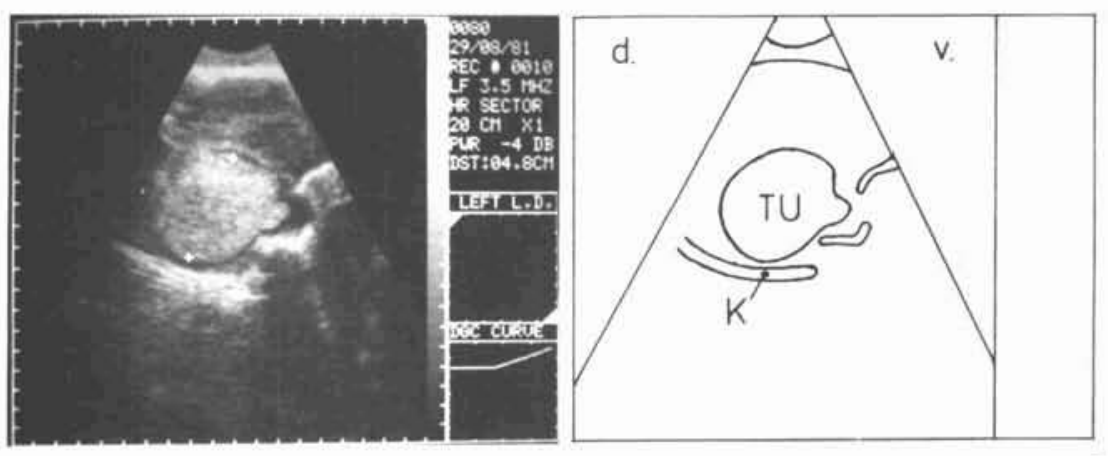

Abb. 1 Parasagittalschnitt Patient 1: Echodichter, gut von der Umgebung abgrenzbarer Tumor (Kreuze) in der hinteren Schädelgrube: $T u=$ Tumor: $\mathrm{K}=$ Schädelkalotte (Os oczipitale) $; \mathrm{d}=$ dorsal; $v=$ ventral.
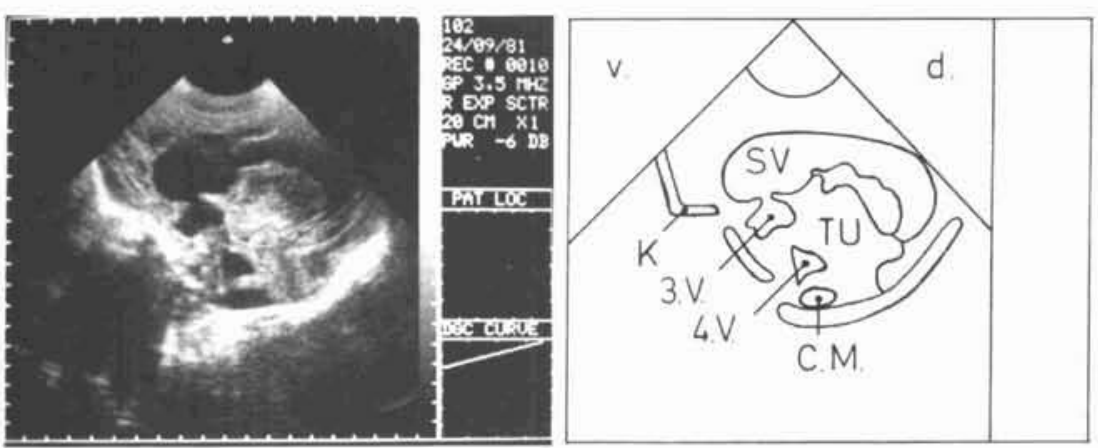

Abb. 2 Medianer Sagittalschnitt Patient 1: Echodichter Tumor in der hinteren Schädelgrube. Tumor von Kleinhirn nicht abgrenzbar. Erweitertes Ventrikelsystem. $T U=$ Tumor $; \mathrm{SV}=$ Seitenventrikel; 3. $V .=3$. Ventrikel; $4 . V .=4$. Ventrikel: $C M=$ Cisterna magna; $K=$ Schädelkalotte (Os frontale) $d=$ dorsal; $v=$ ventral. 

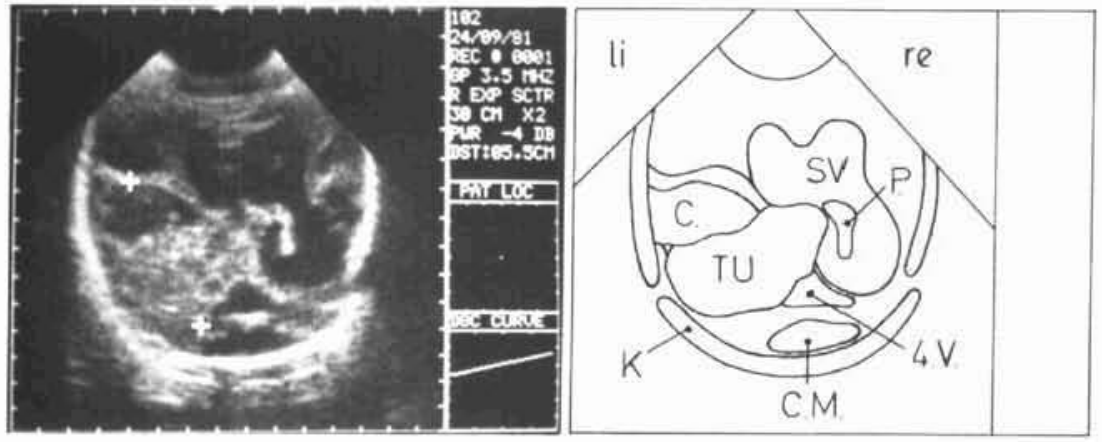

Abb. 3 Nach okzipital gekippter Koronarschnitt Patient 1: Echodichter, okzipital lokalisierter Tumor (Kreuze), der bis zur Mittellinie vorgewachsen ist. Hydrozephalus int. mit zystisch-wabiger Umwandlung beider Großhirnhemisphären. TU = Tumor; $\mathrm{SV}=$ Seitenventrikel; $\mathbf{P}=$ Plexus chorioideus; $4 . V=4$. Ventrikel; $C=$ porenzephale Zyste; $\mathrm{CM}=$ Cisterna magna $\mathrm{K}=$ Schädel. kalotte (Os okzipitale); $1 \mathrm{i}=$ links; $r$ e $=$ rechts

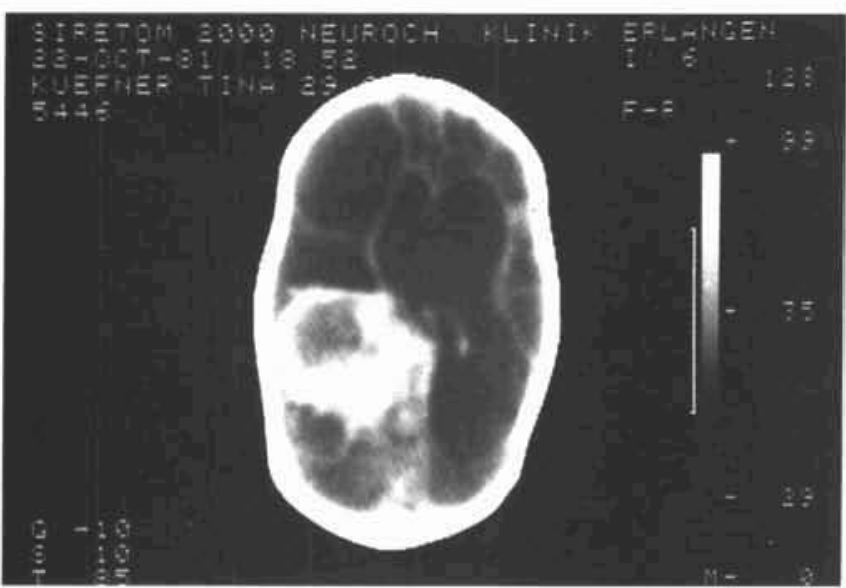

Abb. 4 Computertomogramm Patient 1: Linksseitiger, okzipital lokalisierter Tumor; Hydrozephalus internus mit zystisch-wabiger Umwandlung beider Großhirnhemisphären.

tenventrikels mit zystisch-wabiger Degeneration beider Großhirnhemisphären.

Histologische Diagnose: Unklassifizierbarer, kongenitaler, neuroektodermaler Tumor.

Patient 2 (H. B.): Spontangeburt nach unauffälliger Schwangerschaft. Apgarscore 9/10/10. Geburtsgewicht $3990 \mathrm{~g}$, Kopfumfang $39 \mathrm{~cm}$ (oberhalb der 97. Percentile). Wegen progredienter Zunahme des Kopfumfangs um täglich $1 \mathrm{~cm}$ wurde in einer auswärtigen Klinik ein Computertomogramm durchgefuhrt und die Diagnose Hydrozephalus internus bei angeborener Aquäduktstenose gestellt. Implantation eines ventrikuloperitonealen Shunts im Alter von 7 Tagen. In der
Folgezeit unbeeinflußbare tonisch-klonische Krampfanfälle, Tetraspastik und statomotorische Retardierung. Im Alter von 4 1/2 Monaten stationäre Aufnahme wegen Inappetenz, Erbrechen und zunehmender Müdigkeit.

Befund: Tastbarer, derber Abdominaltumor im rechten Ober- und Mittelbauch, der von der Leber nicht sicher abgrenzbar ist. Makrozephale Kopfform, Kopfumfang $47 \mathrm{~cm}$ (97. Percentile). Fehlende Fixation mit lichtstarrer linker Pupille. Abdomenübersicht, Sonographie und Computertomographie bestätigen die Verdachtsdiagnose eines intraabdominellen Tumors, der sich histologisch als Rhabdoidtumor der rechten Niere erwies.

\section{Diagnostik der intrazerebralen Raumforderung}

a Sonographie: Echodichter, infratentorieller Tumor im Bereich der hinteren Schädelgrube, der bis in den 3. Ventrikel vorgewachsen ist (Abb. 5 und 6). Durchmesser der Raumforderung $8 \mathrm{~cm}$. Massiver Hydrozephalus internus mit Erweiterung beider Seitenventrikel (Abb. 5) und des 3. Ventrikels (Abb, 6). 4. Ventrikel, Kleinhirn und Hirnstamm lassen sich nicht sicher vom Tumor abgrenzen (Abb. 6).

b Computertomographie: Strukturdichter, infratentorieller Tumor, der sich bis zum 3. Ventrikel erstreckt. Tumorinfiltration des Stammhirns sowie des Kleinhirns bis zur Pinealisregion. Erweiterung beider Seitenventrikel und des 3. Ventrikels.

Verlauf: Aufgrund der Größe und Lokalisation des Tumors kam eine operative Freilegung nicht in Frage. Rasche Verschlechterung und Tod durch zentrales Regulationsversagen 1 Woche nach der stationären Aufnahme im Alter von 5 Monaten.
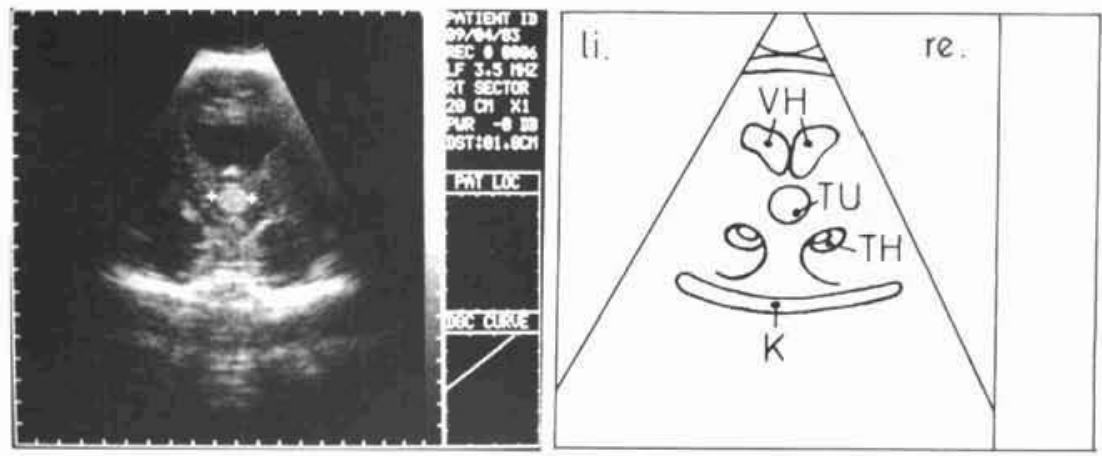

Abb. 5 Koronarschnitt Patient 2: Echodichter. gut von der Umgebung abgrenzbarer Tumor (Kreuze) im Bereich des 3. Ventrikels. Hydrozephalus internus. $T U=$ Tumar; $\mathrm{VH}=$ Vorderhorn: $\mathrm{TH}=$ Temporalhorn: $\mathrm{K}=$ Schädelkalotte (Os okzipitale); $\mathrm{Ii}=$ links; $r$ e $=$ rechts 

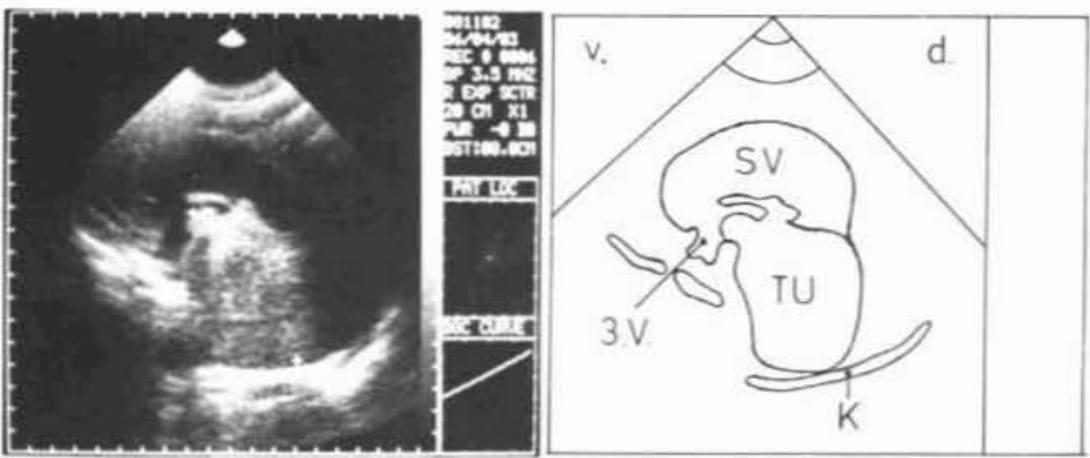

Abb. 6 Medianer Sagittaischnitt Patient 2: Echo. dichter, okzipital lokalisierter Tumor (Kreuze). Ausdehnung bis in den 3 . Ventrikel, von Kleinhirn u. Hirnstamm nicht abgrenzbar. $T U=$ Tumor; $3 . V .=$ 3. Ventrikel; $S V=$ Seitenventrikel; $K=$ Schädelkalotte (Os okzipitale): $v=$ ventral, $d=$ dorsal.
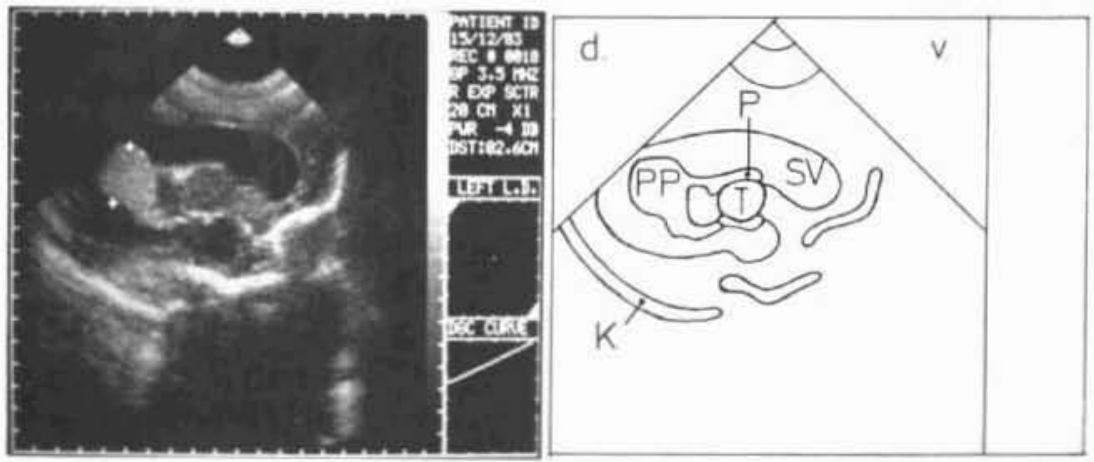

Abb. 7 Parasagittaischnitt Patient 3: Echodichter Tumor im Hinterhorn des rechten Seitenventrikels (Kreuze), der mit dem Plexus kommuniziert und bei der Realtime-Untersuchung pulsierte (Plexuspapillom): Hydrozephalus int. mit Erweiterung des gesamten Seitenventrikels. PP $=$ Plexuspapillom: $\mathrm{P}=$ Plexus chorioideus; $\mathrm{T}=$ Thalamus: $\mathrm{SV}=$ Seiten ventrikel: $K=$ Schadelikalotte (Os okzipitale).

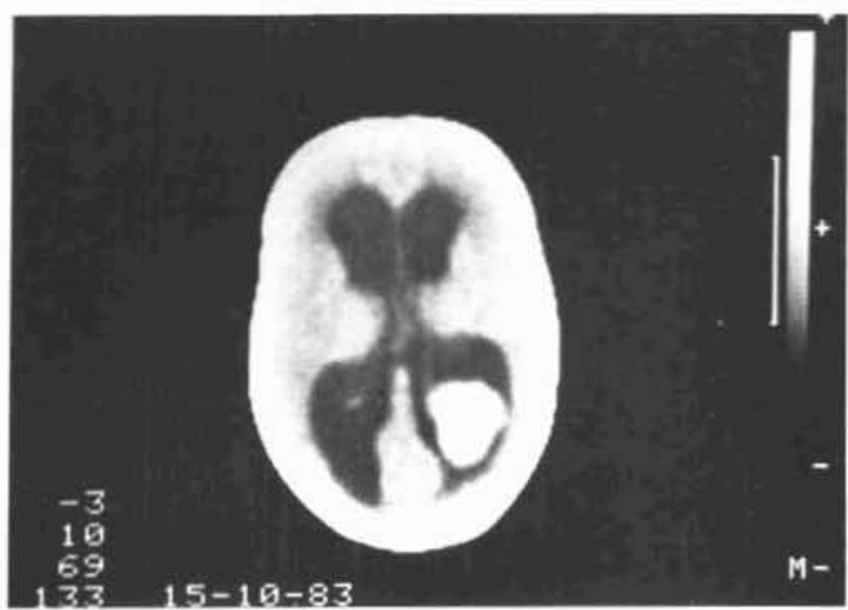

Abb. 8 Computertomogramm Patient 3: Plexuspapillom im Hinterhorn des rechten Seitenventrikels. Hydrozephalus internus.

Sektionsbefund: Tumor des Kleinhimwurmes, der beide Kleinhirnhemisphären und den Hirnstamm im Bereich der Medulla oblongata bis zur Pons cerebri infiltriert hat. Aquäduktstenose mit massiver Dilatation beider Seitenventrikel und des 3 . Ventrikels.

Histologische Diagnose: Gering differenziertes, kongenitales Medulloblastom.

Patient 3 (Sch. St.): Geburt nach unauffalliger Schwangerschaft 1 Woche nach dem errechneten Termin. Ab der 4. Woche hảufiges Erbrechen. Mit 8 Wochen stationäre Aufnahme wegen eines ausgeprägten Sonnenuntergangsphänomens und gespannter, vorgewölbter Fontanelle.

\section{Diagnostik}

a Sonographie: Echodichter, frei im Ventrikellumen flottierender, pulsierender Tumor, der mit dem Plexus chorioideus kommuniziert und im Hinterhorn des rechten Seitenventrikels liegt (Abb. 7). Durchmesser des Tumors $3 \mathrm{~cm}$. Symmetrischer Hydrozephalus internus mit Erweiterung aller Ventrikel.

b Computertomographie: Im Hinterhorn des rechten Seitenventrikels lokalisierter, strukturdichter Tumor

(Abb. 8). Symmetrischer Hydrozephalus internus.

Verlauf: Nach Resektion des Tumors spontane Rückbildung des Hydrozephalus ohne Einlegen einer liquorableitenden Drainage.

Histologische Diagnose: Plexuspapillom.

\section{Diskussion}

Bei den drei Kindern konnte der Hirntumor sonographisch sicher nachgewiesen werden. Vergleiche mit der Computertomographie bzw. mit dem Autopsiebefund zeigten, daß die Sonographie Lokalisation, Gröte und Ausdehnung der Tumore exakt erfaßt hatte. Die Tumore stellten sich im Ultraschall echodicht dar und waren gut vom umgebenden, echoarmen Gehirn und vom echofreien Ventrikellumen abgrenzbar.

Differentialdiagnostisch müssen vor allem Hirnblutungen abgegrenzt werden, die jedoch aufgrund der Lokalisation der Tumor bzw. der Pulsation des Plexuspapilloms bei Patient 3 ausgeschlossen werden konnte. Von anderen Autoren beschriebene zystische Veränderungen der Hirntumoren im Säuglingsalter konnten wir nur bei unserem ersten Patienten feststellen (13). Bereits zum Zeitpunkt 
der Diagnosestellung lag bei allen Kindern ein ausgeprägter Hydrozephalus internus vor. Verlaufskontrollen zeigten bei den beiden inoperablen Kindern die fortschreitende Größenzunahme des Tumors mit Blockade der liquorableitenden Wege und die Entwicklung eines Hydrozephalus okklusivus. Kinder mit einem Plexuspapillom fallen in der Regel durch den Hydrozephalus hypersekretorius auf.

Hirntumore im Säuglingsalter sind sehr selten. Unter 810 histologisch gesicherten, kindlichen Tumoren des Zentralnervensystems betrug die relative Häufigkeit von Hirntumoren im Säuglingsalter 4,3\% (17). Histologisch handelte es sich vorwiegend um neuroepitheliale Tumore $(45,6 \%)$ (17), wobei die häufigsten Hirntumore im Säuglingsalter nach Jänisch (16) Plexuspapillome (19,5\%), Astrozytome $(12,9 \%)$, Medulloblastome $(9,6 \%)$, Ependymome $(6,4 \%)$ und Teratome (13,6\%) sind. Bei Patient 2 wurde neben dem Medulloblastom ein Rhabdoidtumor der rechten Niere gefunden. Bei letzterem handelt es sich um eine sehr seltene Variante des Wilmstumors mit einer weitaus schlechteren Prognose. Die Rhabdoidtumore metastasieren häufig in das Zentralnervensystem. Schmidt u. Mitarb. berichten außerdem über unabhängige $Z$ weittumore in der hinteren Schädelgrube (23), wie bei unserem Patienten:

Bei allen 3 Kindern traten Symptome entweder sofort nach der Geburt oder innerhalb der Neonatalperiode auf, so daß wahrscheinlich in allen 3 Fällen ein angeborener Hirntumor vorlag. Da Hirntumore im Säuglingsal ter meist unspezifische Symptome, wie einen rasch wachsenden Kopfumfang (10, $15,24)$, Erbrechen $(15,24)$, zerebrale Krampfanfälle (15, 24), Verhaltensstörungen (15) und pathologische ophthalmologische Befunde wie Anisokorie, lichtstarre Pupille, fehlende Fixation oder Sonnenuntergangsphänomen verursachen, kommt der frühen Diagnose eine besondere Bedeutung zu. Dabei ist die intrakranielle Sonographie im Säuglingsalter die Methode der Wahl, mit der nicht invasiv, ohne ionisierende Strahlung, Kontrastmittel oder Sedierung des Kindes am Krankenbett untersucht werden kann. Voraussetzung ist ein Sektorscan-Gerät, das bei kleiner Ankoppelungsfläche einen großen Bildausschnitt ermöglicht, womit vor allem zentral gelegene Hirnareale, wie das Ventrikelsystem, die Stammganglien und der Hirnstamm sowie das Kleinhirn exakt erfaßt werden können. Hirntumore, die zu $52 \%$ infratentoriell wachsen (17), lassen sich somit sonographisch gut nachweisen, sofern sie größer als $5 \mathrm{~mm}$ im Durchmesser sind und ein Impedanzunterschied zum umgebenden Gehirn besteht. Schwierigkeiten bereiten evtl. hochparietal oder weit frontal lokalisierte Tumore, die sich bei sehr kleiner Fontanelle (minimaler Durchmesser $1 \mathrm{~cm}$ ) dem sonographischen Nachweis entziehen können. Okzipitale, temporale, zentrale und basisnahe Raumforderungen liegen jedoch im Bereich des Abbildungssektors und sind echographisch diagnostizierbar.

Selbst wenn sich der Tumor, beispielsweise bei sehr kleiner Fontanelle, dem sonographischen Nachweis entziehen sollte, kann zumindest der in einem hohen Prozentsatz vorliegende Begleithydrozephalus erfaßt werden und die weitere Diagnostik mit der CT beschleunigen.

Aufgrund der geschilderten Nachteile wird die Sonographie von ZNS-Tumore wahrscheinlich nicht ganz den von der CT gesetzten Standard erreichen können. So konnten Kazner und Kretzschmar (19) bei 260 CT-Untersuchungen von
Hirntumoren die Raumforderung in $94,6 \%$ der Fälle bei der Erstuntersuchung und in weiteren $4,6 \%$ bei der $Z$ weituntersuchung mit Kontrastmittel nachweisen. Bei der geringen Fallzahl von 3 Patienten ist eine diesbezügliche Aussage über einen Vergleich mit der Sonographie nicht möglich. Bei den 3 vorgestellten Patienten hatten wir jedoch keine Schwierigkeiten, die Lokalisation der Tumore, ihre Größe und Abgrenzbarkeit sowie auch wesentliche Binnenstrukturen sonographisch nachzuweisen.

Weitere diagnostische Hilfsmittel zum Nachweis intrakranieller Raumforderungen im Säuglingsalter, wie die Ventrikulographie, Pneumenzephalographie, Szintigraphie und die Angiographie sind nachzuordnende Methoden. Insbesondere bringt die Szintigraphie im Vergleich zur Computertomographie und zur Sonographie ein wesentlich geringeres Auflösungsvermögen (12). Ventrikulographie und Pneumenzephalographie sind für die Säuglinge belastende Untersuchungsverfahren, deren diagnostischer Wert jetzt von der Sonographie übernommen wurde (30). Die Angiographie hat jedoch nach wie vor präoperativ ihren Platz, um die Lage von Hirntumoren zu den Hirngefảßen und die Tumorgefäße selbst darzustellen.

Die kraniale Sonographie ist somit heute in der Diagnostik intrakranieller Raumforderungen des Neugeborenen und Säuglings die Methode der ersten Wahl. Auch wenn aufgrund ihrer Wachstumspotenz diese gering differenzierten Hirntumore des frühen Kindesalters quoad vitam eine schlechte Prognose zeigen, bringt die Sonographie bei Plexuspapillomen als häufigsten Geschwülsten dieser Altersgruppe einen Zeitgewinn für die chirurgische Therapie. In der Zukunft ließe sich somit die sekundäre Druckschädigung des Gehirns einschränken. Pulssynchrone Echoänderungen und die Plexus-bezogene Lage des Tumors erlauben in Einzelfällen die sonographische Artdiagnose. Aufgrund unserer Erfahrung gilt diese Feststellung nur für das Plexuspapillom.

\section{Literatur}

1 Allan, W.C., P.J. Holt, L.R. Sawyer, A.M. Tito, S.K. Meade: Ventricular dilatation after neonatal periventricular-intraventricular hemorrhage. J. Dis. Child. 136 (1982) 589-593

2 Altenburg, H., M. Brandt, H.J. König: Ventriculography as a diagnostic procedure in CNS tumours in childhood: Its indications and interpretation. In: Voth, D., P. Gutjahr, C. Langmaid: Tumours of the central nervous system in infancy and childhood. Springer, Berlin, Heidelberg, New York (1982)

3 Bejar, R., V. Curbelo, R.W. Coen, G. Leopold, H. James, L. Gluck: Diagnosis and follow-up of intraventricular and intracerebral hemorrhages by ultrasound studies of infant's brain through the fontanelles and sutures. Pediatrics 66 (1980) $661-673$

4 Bliesner, J.A.: Intrakranielle Veränderungen im Säuglings- und frühen Kindesalter. Technik und Ergebnisse der Sonographie. Monatsschr. Kinderheilkd. 129 (1981) 200-219

5 Deeg, K.H., W. Dick, V. Spitzer, K. Richter: Sonographische Verlaufskontrolle der fortschreitenden Ventrikelerweiterung beim posthämorrhagischen Hydrozephalus. In: Voth, $D$., $P$. Gutjahr, $P$. Glees: Hydrozephalus im frühen Kindesalter. Enke, Stuttgart (1983)

6 Deeg, K.H., W. Dick, V. Spitzer, K. Richter: Früherkennung des posthämorrhagischen Hydrozephalus mit Hilfe der Sonographie. Monatsschr. Kinderheilkd. 130 (1982) 657

7 Deeg, K.H., K. Richter, K. Stehr: Sonographischer Nachweis von Hirnblutungen beim Frühgeborenen. Klin. Pädiat. 195 (1983) 299 
8 Dewbury, K.C., A.P.R. Aluwihare: The anterior fontanelle as an ultrasound window for the study of the brain: A preliminary report. British J. Radiol. 53 (1980) 81-84

9 Dubowitz, L.M.S., M.I. Levene, A. Morante, P. Palmer, V. Dubowitz: Neurologic signs in neonatal intraventricular hemorrhage: A correlation with real-time ultrasound. J. Ped. 99 (1981) $127-133$

10 Gerlach, H., W. Jänisch, D. Schreiber: Intracranial and Spinal tumours in newborns and infants. In: Voth, D., P. Gutjahr, C. Langmaid: Tumours of the central nervous system in infancy and childhood. Springer, Berlin, Heidelberg, New York (1982)

11 Haber, K., R.D. Wachter, P.C. Christenson, Y. Vaucher, D.J. Sahn: Ultrasonic evaluation of intracranial pathology in infants. A new technique. Radiology 134 (1980) 173-178

$12 \mathrm{Hahn}, \mathrm{K}$ : Radionuclide imaging of cerebral tumours in children. In: Voth, D., P. Gutjahr, C. Langmaid: Tumours of the central nervous system in infancy and childhood. Springer, Berlin, Heidelberg, New York (1982)

13 Han, B.K., D.S. Babcock, A.E. Oestreich: Sonography of brain tumors in infants. Pediatr. Radiol. 14 (1984) 183

14 Horbar, J.D., C.L. Walters, A.G.S. Philip, J.F. Lucey: Ultrasound detection of changing ventricular size in posthemorrhagic hydrocephalus. Pediatrics 66 (1980) 674-678

15 Jakobi, G.: Clinical presentation of space-occupying lesions of the central nervous system. In: Voth, D., P. Gutjahr, C. Lang. maid: Tumours of the central nervous system in infancy and childhood. Springer, Berlin, Heidelberg, New York (1982)

16 Jänisch, W., D. Schreiber, H. Gerlach: Tumoren des Zentralnervensystems bei Feten und Säuglingen. Fischer, Jena (1980)

17 Jellinger, $K$., E. Machacek: Rare intracranial tumours in infancy and childhood. In: Voth, D., P. Gutjahr, C. Langmaid: Tumours of the central nervous system in infancy and childhood. Springer, Berlin, Heidelberg, New York (1982)

18 Jellinger, K., M. Sunder-Plassmann: Connatal intracranial tumours. Neuropädiatrie 4 (1973) 46-63

19 Kazner, E., K. Kretzschmar: Computer tomography diagnosis of CNS tumours in childhood. In: Voth, D., P. Gutjahr, C. Langmaid: Tumours of the central nervous system in infancy and childhood. Springer, Berlin, Heidelberg, New York (1982)

20 Lipscombe, A.P., R.J. Blackwell, E.O.R. Reynolds, R.J. Thorburn et al.: Ultrasound scanning of brain through anterior fontanelle of newborn infants. Lancet 2 (1979) 39
21 Morgan, C.L., W.S. Trought, S.J. Rothman, J.P. Jimenez: Comparison of grey-scale ultrasonography and $C T$ in the evaluation of macrocrania in infants. Radiology 132 (1979) 119-123

22 Ondrus, B., J. Babala, L. Hostyn: Tumours of children till one year of age. Neoplasma 12 (1965) 323-332

23 Schmidt, D., D. Harms, G. Zieger: Malignant rhabdoid tumor of the kidney. Histopathology, ultrastructure and comments of differential diagnosis. Virchows Arch. 398 (1982) $101-$ 108

24 Schreiber, D., W. Jänisch, H. Gerlach: CNS tumours in infancy, childhood and adolexcence. In: Voth, D., P. Gutjahr, C. Lang. maid: Tumours of the central nervous system in infancy and childhood. Springer, Berlin, Heidelberg, New York (1982)

25 Silverboard, G., M.H. Holder, P.A. Ahmann, A. Lazarra, J.F. Schwartz: Reliability of ultrasound in diagnosis of intracranial hemorrhage and posthemorrhagic hydrocephalus: Comparison with computed tomography. Pediatrics 66 (1980) 507-514

26 Sivakoff, M., S. Nouri: Diagnosis of vein of Galen arteriovenous malformation by two-dimensional ultrasound and pulsed doppler method. Pediatrics 69 (1982) 84-86

27 Skolnick, M.L., A.E. Rosenbaum, T. Matzuk, A.N. Guthkelch, E.R. Heinz: Detection of dilated cerebral ventricles in infants: A correlative study between ultrasound and computed tomography. Radiology 131 (1979) 447-451

28 Straßburg, H.M., M. Sauer: Morphologische Darstellung und Identifizierung eines Aneurysmas der Vena Galeni beim Säugling mit der Duplex-Scan-Technik. Klin. Pädiatr. 194 (1982) $84-87$

29 Sunder-Plassmann, M., V. Grunert: Fehldiagnosen bei konnatalen Hirntumoren. Z. Kinderchir. 8 (1970) 352-358

30 Wende, S., ?. Kishikawa, N. Hüwel, E. Kazner, Th. Grumme, W.R. Lanksch: Computed tomography and/or ventriculography? In: Voth, D., P. Gutjahr, C. Langmaid: Tumours of the central nervous system in infancy and childhood. Springer, Berlin, Heidelberg, New York (1982)

31 Wiesemann, H.G.: Intrakranielles Teratom bei einem Neugeborenen. Pädiat. prax. 21 (1979) 27-30

Dr. K.H. Deeg, Universitätskinderklinik, Loschgestr. 15, D-8520 Erlangen

Dr.H. Segerer, Universitätskinderklinik, Loschgestr. 15, D-8520 Erlangen

Prof. Dr. D. Harms, Universitätskinderklinik, Loschgestr. 15, D-8520 Erlangen 